\title{
Awareness of illness and suicidal behavior in delusional disorder patients
}

\author{
Oriol Molina-Andreu1, Alexandre González-RodrígueZ², AleXandre Pons Villanueva², Rafael Penadés², \\ Rosa Catalán', Miquel Bernardo2
}

1 Department of Mental Health, Hospital Universitari Mútua de Terrassa, Spain.

2 Schizophrenia Unit. Clinical Institute of Neurosciences. Hospital Clinic, University of Barcelona, Spain.

Received: 5/22/2014 - Accepted: 9/15/2014

DOI: 10.1590/0101-60830000000034

\begin{abstract}
Background: The relationship between insight and suicidal behavior among psychotic patients is poorly studied and possibly mediated by clinical variables. Objectives: Our goal was to investigate clinical differences in suicidal and non-suicidal delusional disorder (DD) patients, and to evaluate the relationship between insight, psychotic and depressive symptoms. Methods: Cross-sectional study in 64 consecutive DD patients. For assessment, we used the Positive and Negative Syndrome Scale (PANSS), the Hamilton Rating Scale for Depression (HRSD-17), the Columbia Suicide Severity Rating Scale (C-SSRS), the Personal and Social Performance Scale (PSP), and the first three items of the SUMD scale for insight. The sample was divided according to the presence of suicide attempts. To investigate psychopathological associations, bivariate correlation coefficients were used. Age at onset served as covariate in subsequent analyses. Results: Suicidal DD patients had higher depressive symptoms and were more frequently admitted than non-suicidal patients. A logistic regression model confirmed that insight, depressive symptoms and age at onset were predictors of suicidal behavior. Unawareness of the effects of medication was negatively related to depressive symptoms. After adjustment, depressive symptoms were weakly correlated to better insight into the effects of medication. No other statistically significant correlations were found. Discussion: Depressive symptoms, insight and age at onset of disease may be potential predictors of suicidal behavior in DD patients.
\end{abstract}

Molina-Andreu O et al. / Arch Clin Psychiatry. 2014;41(6):156-8

Keywords: Psychosis, insight, delusional disorder, paranoia, suicide, suicidal behaviour, psychopathology.

\section{Introduction}

For many decades, poor insight has been considered a prevalent feature in patients diagnosed with chronic psychotic disorders ${ }^{1}$. The vast majority of scientific literature has defined insight as a multidimensional concept that includes awareness of a mental illness, recognition of the need for medication, awareness of the social consequences, and awareness of the presence of psychotic symptoms and the attribution of symptoms to the illness ${ }^{2}$.

Suicide attempts before first psychiatric consultation are prevalent in psychotic patients and associated with depressive comorbidity ${ }^{3,4}$, and insight has also been related to suicidal behavior rates ${ }^{5}$. Several studies have indicated that high levels of insight, or some aspects of insight, may increase the risk for suicidal behavior ${ }^{5}$; on the other hand, other authors have found no relationships between insight and suicidal ideation or behavior in first-episode of psychosis

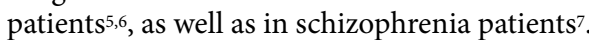

A recent systematic review reported that the association between suicidal behavior or ideation and insight may be mediated by other clinical variables such as depressive symptoms ${ }^{2}$. On the other hand, the authors hightlighted that inconsistency of findings regarding the influence of insight on suicidal behaviour may be explained by some methodological problems identified in the reviewed studies. However, this subject has been poorly studied in delusional disorder (DD) patients.

The main aims of this study were to investigate clinical differences in suicidal and non-suicidal DD patients, and to examine the relationship between insight, psychotic and depressive symptoms, as this topic has been shown to be of clinical interest in delusional patients.

\section{Methods}

\section{Study design and participants}

We carried out a cross-sectional study by including all consecutive cases of DD outpatients who attended the Barcelona Clinic Schizophrenia Unit (Barcelona, Spain), between 2008 and 2013. All patients fulfilled the following inclusion criteria: (a) diagnosis of DD according to DSM-IV-TR criteria, (b) age over 18 years, and (c) not having a previous diagnosis of schizophrenia, mental retardation or organic psychosis.

The present study is a part of an ongoing study on schizophrenia and related disorders, which was approved by the Ethics Committee of the Hospital Clinic (Ref. 2007/3699).

\section{Assessment measures}

The diagnosis of DD was confirmed by using the psychosis module of the Structured Clinical Interview for DSM-IV axis I disorder, clinical version (SCID-I CV), which was assessed by two senior psychiatrists in charge of the cases.

We used the Positive and Negative Syndrome Scale 8 to assess positive, negative and general psychotic symptoms. Depressive symptoms were assessed by using the Spanish version of the 17-item Hamilton Rating Scale for Depression (HRSD) ${ }^{9}$, and functionality by the Personal and Social Performance Scale (PSP) ${ }^{10}$. Furthermore, the severity of suicidal ideation and number of suicide attemps were obtained by using the Columbia Suicide Severity Rating Scale (C-SSRS $)^{11}$. The first three items of the Scale to Assess Unawareness of Mental Disorder (SUMD) ${ }^{12}$ Spanish Version, were used for assessing awareness of illness, awareness of the need for medication and social consequences. The SUMD scale has shown a good reability and validity, and ranges from 1 to 5 in which higher scores are indicative of unawareness of the multiple components.

Mean scores of the assessment scales were considered outcome variables, and sociodemographic and other clinical data served as secondary outcomes. The sample was divided into two groups according to the presence or absence of suicidal attempts at lifetime.

\section{Statistical analysis}

All data were analyzed by using SPSS for Windows (Version 19.9; SPSS Inc., Chicago, Illinois, USA). Univariate differences in sociodemographic and clinical characteristics, and assessment scales between patients who attempted suicide and those who were not attempters were tested by using Mann-Whitney $U$ test, $\chi 2$ and Fisher's exact tests, as the distribution of data were non-parametric. A logistic 
regression analysis was conducted to identify potential predictors of suicidal behavior in DD patients using depressive symptoms (HRSD-17), age at onset of DD, social network, and SUMD total scores. The relationship between the three first items of the SUMD scale and insight total scores, psychopathological symptoms and functionality were investigated using the Spearman correlation coefficients. In this analysis, the original sample was not divided. In a second step, partial correlation coefficients were applied with age at onset of DD as confounding variable. Significance level was set at 0.05 (two-tailed).

\section{Results}

\section{Sociodemographic and clinical features in suicidal and non-suicidal DD patients}

Of the $64 \mathrm{DD}$ patients included in the study, $10(15.6 \%)$ had attempted suicide at lifetime. No statistically significant differences were found with regard to sociodemographic variables between $\mathrm{DD}$ patients who attempted suicide and those who did not attempt.

Suicidal DD patients showed higher rates of depressive comorbidity $(p=0.025)$, were more likely to receive antidepressants $(p=0.011)$ and were more frequently admitted to a psychiatric ward $(\mathrm{p}=0.026)$ than non-suicidal patients.

Regarding insight and psychopathological symptoms, suicide attempters showed higher depressive symptoms measured by the HRSD-17 scale ( $\mathrm{p}=0.011)$, higher severity of suicidal ideation $(\mathrm{p}<$ 0.001 ) and less awareness of social consequences with higher scores of the SUMD scale item 3 ( $\mathrm{p}=0.022)$.

A logistic regression analysis was conducted to predict suicidal behavior using potential confounders as predictors. The Wald criterion demonstrated that insight $(\mathrm{p}=0.048)$, depressive symptoms (0.004) and age at onset of disease $(\mathrm{p}=0.038)$ made a significant contribution to prediction. Social support as measured by total number of cohabiters was not a significant predictor of suicidal behavior (Table 1).

Table 1. Demographic and clinical data, and assessment scales by clinical groups

\begin{tabular}{|c|c|c|c|c|}
\hline Variables & Total sample $\mathrm{n}=64$ & No suicide attempters $n=54$ & Suicide attempters $n=10$ & Statistic \\
\hline \multicolumn{5}{|l|}{ Sociodemographic measures } \\
\hline Age, mean (SD) & $55.02(12.78)$ & $54.85(13.49)$ & $55.90(8.33)$ & $U(63)=254, Z=-0.269, p=0.767$ \\
\hline Marital status [n(\%)] & & & & $\chi^{2}=0.598, d f=2, p=0.742$ \\
\hline Single & $23(35.9)$ & $20(37.04)$ & $3(30)$ & \\
\hline Married/living together & $16(25)$ & $14(25.93)$ & $2(20)$ & \\
\hline Separated/divorced/widowed & $25(39.1)$ & $20(37.1)$ & $5(50)$ & \\
\hline Educational level, years [n(\%)] & & & & $\chi^{2}=0.238, d f=3, p=0.971$ \\
\hline$<8$ & $7(11)$ & $6(11.11)$ & $1(10)$ & \\
\hline $8-9$ & $16(25)$ & $14(25.93)$ & $2(20)$ & \\
\hline $10-11$ & $22(34.4)$ & $18(33.33)$ & $4(40)$ & \\
\hline 12 or $>$ & $19(29.6)$ & $16(29.63)$ & $3(30)$ & \\
\hline Employment status [n(\%)] & & & & $\chi^{2}=1.392, d f=2, p=0.707$ \\
\hline Unemployed & $4(6.25)$ & $4(7.4)$ & $0(0)$ & \\
\hline Employed & $21(32.81)$ & $18(33.33)$ & $3(30)$ & \\
\hline Economic benefit & $39(60.94)$ & $32(59.26)$ & $7(70)$ & \\
\hline \multicolumn{5}{|l|}{ Clinical measures } \\
\hline Age at onset of DD, mean (SD) & $48.83(12.61)$ & $47.87(13.07)$ & $54(8.5)$ & $U(63)=192, Z=-1.44, p=0.149$ \\
\hline Accumulated years of disease, mean (SD) & $12.25(11.64)$ & $12.78(12.13)$ & $9.4(8.4)$ & $U(63)=241, Z=-0.538, p=0.591$ \\
\hline $\mathrm{DD}$ type $[\mathrm{n}(\%)]$ & & & & $\chi^{2}=2.735, d f=4, p=0.603$ \\
\hline Persecutory & $52(81.25)$ & $42(77.78)$ & $10(100)$ & \\
\hline Erotomanic & $5(7.81)$ & $5(9.26)$ & $0(0)$ & \\
\hline Jealous & $3(4.69)$ & $3(5.56)$ & $0(0)$ & \\
\hline Grandiose & $2(3.13)$ & $2(3.71)$ & $0(0)$ & \\
\hline Somatic & $2(3.13)$ & $2(3.71)$ & $0(0)$ & \\
\hline Depressive comorbidity, [n(\%)] & $22(34.4)$ & $15(27.78)$ & $7(70)$ & $p=0.025^{*} ;$ FET \\
\hline Antidepressant use, [n(\%)] & $26(40.63)$ & $18(33.33)$ & $8(80)$ & $p=0.011^{*} ;$ FET \\
\hline Lifetime admissions, mean (SD) & $1.02(1.13)$ & $0.89(1.09)$ & $1.7(1.16)$ & $U(63)=156.5, Z=-2.22, p=0.026^{*}$ \\
\hline Number of suicide attemps, mean (SD) & $0.23(0.66)$ & $0(0)$ & $1.5(0.972)$ & $U(63)=0.0, Z=-7.913, p=0.000^{*}$ \\
\hline \multicolumn{5}{|l|}{ Assessment scales, mean (SD) } \\
\hline PANSS total scale & $83.83(18.46)$ & 84.04 (17.58) & $82.70(23.74)$ & $U(63)=247, Z=-0.060, p=0.952$ \\
\hline PANSS positive subscale & $22.59(5.83)$ & $23.04(5.85)$ & $20.2(5.33)$ & $U(63)=190.5, Z=-1.472, p=0.141$ \\
\hline PANSS negative subscale & $18.17(5.71)$ & $18.07(5.67)$ & $18.70(6.26)$ & $U(63)=251.5, Z=-0.343, p=0.732$ \\
\hline PANSS general subscale & $43.06(9.2)$ & $42.93(8.41)$ & $43.8(13.24)$ & $U(63)=244, Z=-0.481, p=0.630$ \\
\hline SUMD scale & $14.19(1.47)$ & $14.11(1.54)$ & $14.60(0.97)$ & $U(63)=215, Z=-1.176, p=0.239$ \\
\hline SUMD, awareness illness & $4.81(0.56)$ & $4.81(0.55)$ & $4.80(0.63)$ & $U(63)=268, Z=-0.068, p=0.946$ \\
\hline SUMD, awareness effects of medication & $4.73(0.57)$ & $4.72(0.6)$ & $4.80(0.42)$ & $U(63)=265, Z=-0.132, p=0.895$ \\
\hline SUMD, awareness social consequences & $4.64(0.574)$ & $4.57(0.60)$ & $5(0)$ & $U(63)=170, Z=-2.282, p=0.022^{*}$ \\
\hline PSP score & $52.64(15.26)$ & $52.26(15.91)$ & $54.70(11.55)$ & $U(63)=258, Z=-1.36, p=0.174$ \\
\hline HRSD-17 score & $10.48(6.21)$ & $9.54(5.62)$ & $15.60(7.03)$ & $U(63)=132.5, Z=-2.552, p=0.011^{*}$ \\
\hline Suicidal ideation severity (C-SSRS) & $3.97(7.31)$ & $1.94(4.92)$ & $14.90(8.62)$ & $U(63)=72.5, Z=-4.803, p=0.000^{*}$ \\
\hline
\end{tabular}

$* P<0.05$.

C-SSRS: Columbia Suicide Severity Rating Scale; DD: delusional disorder; df: degrees of freedom; FET: Fisher's Exact Test; HRSD: Hamilton Rating Scale for Depression; p: p-value; PANSS: Positive and Negative Syndrome Scale; PSP: Personal and Social Performance Scale; SD: Standard deviation; SUMD: total for items 1, 2 and 3; U: Mann-Whitney U test; $\chi^{2}$ : Chi-square test. 


\section{Correlational analysis between insight and psychopathological symptoms}

SUMD total score was negatively associated with the age at the study inclusion $(\mathrm{r}=0.335, \mathrm{p}=0.007)$ and age at onset of DD $(\mathrm{r}=-0.375$, $\mathrm{p}=0.002)$.

Prior to the correction by potential confounding factors, although no statistical significance was reached, total insight showed a tendency toward a positively correlation with accumulated years of disease $(r=0.227, p=0.072)$ and was negatively related to PANSS general subscale scores $(r=-0.225, p=0.073)$. SUMD total scores were not related to positive and negative symptoms, functionality, depressive symptoms or severity of suicidal ideation, as measured by the C-SSRS scale. Unawareness of disease and of the effects of medication showed a tendency to be negatively associated with depressive symptoms $(\mathrm{r}=-0.215, \mathrm{p}=0.088 ; \mathrm{r}=-0.235, \mathrm{p}=0.062)$. Unawareness of the social consequences was negatively associated with PANSS total scale $(\mathrm{r}=-0.266, \mathrm{p}=0.034)$, PANSS general subscale $(\mathrm{r}=-0.258$, $\mathrm{p}=0.040)$, current age $(\mathrm{r}=-0.344, \mathrm{p}=0.005)$ and age at onset of $\mathrm{DD}(\mathrm{r}=-0.368, \mathrm{p}=0.003)$, and positively associated with number of suicide attempts $(\mathrm{r}=0.287, \mathrm{p}=0.022)$. A tendency toward positively correlation was shown between unawareness of social consequences and functionality $(\mathrm{r}=0.235, \mathrm{p}=0.061)$.

In a second step, age at onset of DD was included into the correlational analysis as confounding variable. After adjustment, unawareness of the effects of medication was negatively related to depressive symptoms $(\mathrm{r}=-0.264, \mathrm{p}=0.037)$. Other correlations were no longer statistically significant.

\section{Discussion}

In our sample, suicidal delusional disorder (DD) patients had higher depressive symptoms and were more frequently admitted to a psychiatric ward than non-suicidal patients. These findings are consistent with a previous study carried out by our team ${ }^{4}$. In the aforementioned study we found that DD patients with depressive comorbidity (DC) had higher rates of suicidal behavior and suicidal ideation than patients without DC. Thus, based on our findings, it is not possible to exclude a relationship between insight and suicidal behavior in DD patients, as suggested by some authors ${ }^{7}$.

In the present study, after conducting regression models, depressive symptoms, insight and age at onset of disease were confirmed to be clinical predictors of suicidal behavior in DD patients. Further, this is in agreement with a recent systematic review carried by LópezMorínigo and coworkers ${ }^{2}$, who concluded that if any relationship exists between insight and suicidal behavior in psychotic patients, it may be related to other clinical variables, such as depressive symptoms.

In a further step, we carried out a correlation analysis between total insight scores, the first three items of the SUMD scale, and psychopathological symptoms. When uncorrected for potential confounders, DD patients with high clinical insight in terms of awareness of disease and recognition of the effects of medication had significantly higher depression scores and greater histories of suicide attempts compared to patients with low clinical insight. Although statistical significance was not reached, these findings are in agreement with the systematic review mentioned above ${ }^{2}$. Furthermore, patients with better awareness of the social consequences had a later age at onset of DD.

In a second step, we included age at onset of DD into the correlational analysis as confounding variable, as it was significantly correlated with insight scores in the non-controlled correlation analysis. When adjusted, DD patients with higher depressive symptoms showed better insight into the effects of medication, which was probably mediated by age at onset of DD. In our point of view, it is reasonable that patients with an earlier psychotic diagnosis would show lower insight levels than patients with a later age at onset.
To the best of our knowledge, this is the first study to specifically investigate sociodemographic and clinical differences in two clinical subgroups of DD patients, those who have attempted suicide at lifetime and those who did not attempt it. Furthermore, the main strength of our study is that it is the first to specifically investigate clinical correlations between insight, depressive and psychotic symptoms, and number of previous suicide attempts, specifically in a sample formed by DD patients. Some limitations in our study should be taken into account. Our study has a cross-sectional design and the sample size is small.

\section{Acknowledgments}

This work has been partially supported by a grant for Rosa Catalán from the Fondo de Investigaciones Sanitarias (FIS:040209) and by the Fondo Europeo de Desarrollo Regional (FEDER) "Una manera de hacer Europa". We would like to thank the Government of Catalonia, Comissionat per Universitats I Recerca del Departament d'Innovació, Universitats i Empresa (DIUE) 2009SGR1295, and the Instituto de Salud Carlos III, Centro de Investigación Biomédica en Red de Salud Mental, CIBERSAM, and all staff for their collaboration in completing demographic and clinical data in medical record systems.

\section{Conflicts of interest}

Dr. Miquel Bernardo has received honoraria from Bristol-MeyerSquibb y Wyeth, Janssen-Cilag, Eli Lilly, Pfizer, Synthelab, Glaxo Smith Kline, and Astra-Zeneca; however, these are not related with the content of the manuscript. The remaining authors have no conflicts of interest to declare.

\section{References}

1. Ouzir M, Azorin JM, Adida M, Boussaoud D, Battas O. Insight in schizophrenia: from conceptualization to neuroscience. Psychiatry Clin Neurosci. 2012;66:167-79.

2. López-Morínigo JD, Ramos-Ríos R, David AS, Dutta R. Insight in schizophrenia and risk of suicide: a systematic update. Compr Psychiatry. 2012;53:313-22.

3. González-Rodríguez A, Molina-Andreu O, Navarro V, Gastó C, Penadés R, Catalán R. Delusional disorder: no gender differences in age at onset, suicidal ideation, or suicidal behavior. Rev Bras Psiquiatr. 2014;36(2):119-24.

4. González-Rodríguez A, Molina-Andreu O, Penadés Rubio R, Catalán Campos R, Bernardo Arroyo M. [Clinical significance of suicidal behaviour in delusional disorder: a 44 case-series descriptive study]. Med Clin (Barc). 2014;142(7):299-302.

5. Melle I, Barrett EA. Insight and suicidal behavior in first-episode schizophrenia. Expert Rev Neurother. 2012;12:353-9.

6. Barrett EA, Sundet K, Faerden A, Agartz I, Bratlien U, Romm KL, et al. Suicidality in first episode psychosis is associated with insight and negative beliefs about psychosis. Schizophr Res. 2010;123:257-62.

7. Schwartz RC, Smith SD. Suicidality and psychosis: the predictive potential of symptomatology and insight into illness. J Psychiatr Res. 2004;38:185-91.

8. Kay SR, Fiszbein A, Vital-Herne M, Fuentes LS. The Positive and Negative Syndrome Scale - Spanish adaptation. J Nerv Ment Dis. 1990;178:510-7.

9. Ramos-Brieva JA, Cordero-Villafafila A. A new validation of the Hamilton Rating Scale for Depression. J Psychiatr Res. 1988;22:21-8.

10. Apiquian R, Elena UR, Herrera-Estrella M, Moreno-Gomez A, Erosa S Contreras V, et al. Validity of the Spanish Version of the Personal and Social Performance Scale in schizophrenia. Schizophr Res. 2009;112:181-6.

11. Posner K, Brown GK, Stanley B, Brent DA, Yershova KV, Oquendo MA et al. The Columbia-Suicide Severity Rating Scale: initial validity and internal consistency findings from three multisite studies with adolescents and adults. Am J Psychiatry. 2011;168:1266-77.

12. Ruiz A, Pousa E, Duñó R, Crosas J, Cuppa S, García C. [Spanish adaptation of the Scale to Asses Unawareness of Mental Disorder (SUMD)]. Actas Esp Psiquiatr. 2008;36(2):111-1198. 